\title{
Clinicopathologic implications of the miR-197/PD-L1 axis in oral squamous cell carcinoma
}

\author{
Hyein Ahn ${ }^{1}$, Jeong Mi Yang ${ }^{1}$, Hyojin Kim¹, Jin-Haeng Chung ${ }^{1}$, Soon-Hyun Ahn ${ }^{2}$, \\ Woo-Jin Jeong ${ }^{2}$ and Jin Ho Paik ${ }^{1}$ \\ ${ }^{1}$ Department of Pathology, Seoul National University Bundang Hospital, Seoul National University College of Medicine, \\ Seongnam, Korea \\ ${ }^{2}$ Department of Otorhinolaryngology, Seoul National University Bundang Hospital, Seoul National University College of \\ Medicine, Seongnam, Korea \\ Correspondence to: Jin Ho Paik, email: paikjh@snu.ac.kr
}

Keywords: PD-L1, oral squamous cell carcinoma, miR-197, microRNA, tumor-infiltrating lymphocytes

Received: December 02, $2016 \quad$ Accepted: June 27, $2017 \quad$ Published: August 03, 2017

Copyright: Ahn et al. This is an open-access article distributed under the terms of the Creative Commons Attribution License 3.0 (CC BY 3.0), which permits unrestricted use, distribution, and reproduction in any medium, provided the original author and source are credited.

\section{ABSTRACT}

Immune escape of a tumor from tumor-infiltrating lymphocytes (TILs) is induced by PD-L1, which is suppressed by miR-197. We investigated the clinicopathologic implications of the miR-197/PD-L1 axis and its effects on TILs and the clinicopathologic features of oral squamous cell carcinoma (OSCC). We used RT-PCR and immunohistochemistry in 68 OSCC patients to analyze the correlations between tumoral expression of $\mathrm{miR}-197$ and PD-L1 and the degree of tumoral invasion by TILs (CD3+, CD4+, CD8+, PD-1+, FoxP3+, and CD20+ lymphocytes). PD-L1 levels correlated inversely with miR-197 but correlated positively with TILs. The aggressive features of OSCC, including high stage, angiolymphatic invasion, perineural invasion, and death, were associated with TIL depletion. High T stage (T4) tumors also had low PD-L1 but had high miR-197 expression. In a univariate survival analysis of the full cohort, high miR-197 was associated with poor overall survival, whereas high PD-L1 expression ( $2+$ ) associated with good overall survival. In a multivariate analysis stratified based on miR-197 (median), high PD-L1 expression (2+) was an independent favorable prognostic factor for overall survival $(P=0.040)$ in the miR-197 ${ }^{\text {high }}$ subgroup but not the miR-197low subgroup. These findings may have clinicopathologic implications for the miR-197/PD-L1 axis and TILs in OSCC.

\section{INTRODUCTION}

Oral cancer accounts for $1 \%$ to $5 \%$ of human malignancies, and its occurrence has increased for more than a decade [1]. Oral squamous cell carcinoma (OSCC) is the predominant type and comprises approximately $90 \%$ of all oral cancers [2]. OSCC occurs mostly in males with long-term exposure to tobacco and alcohol who are in their fifth to seventh decade of life [3]. The prognosis of OSCC remains poor because of the tendency of the cancer to metastasize [4]. Despite recent advances in combined surgery, chemotherapy, and radiotherapy, the survival rates of patients with OSCC has shown no improvement over the past few decades [2]. Therefore, an understanding of the molecular mechanism underlying progression of OSCC in the context of the tumor microenvironment is needed for improvement of the therapeutic strategies for the disease.

Programmed cell death ligand-1 (PD-L1) is a cell-surface glycoprotein that induces $\mathrm{T}$ cell anergy and apoptosis by activating the PD-1 receptors on T lymphocytes [5]. Normally, PD-L1 is a factor in maintaining immunologic homeostasis, but in many cancers PD-L1 is overexpressed on tumor cells as well as on subsets of immune cells, including T cells, B cells, macrophages, and dendritic cells. Blocking the PD- 
L1/PD-1 pathway reverses the immune escape of the tumor and improves the anticancer immune responses in microenvironments containing tumor-infiltrating lymphocytes (TILs) [6]. PD-L1 has gained attention for its benefits in clinical trials of cancer immunotherapy. However, the number of studies on the biologic mechanisms and effects of PD-L1 in OSCC is limited $[7,8]$, and the clinicopathologic implications, including prognostic values and associations with TILs, have not been clarified.

MicroRNAs (miRNAs) are single-stranded, noncoding short RNAs consisting of 20 to 22 nucleotides that inhibit the translation of genes by disrupting specific messenger RNAs. In recent studies, the expression profiles of miRNAs in OSCC were examined and some miRNAs were found to correlate with tumorigenesis and cancer progression [9, 10]. MiR-197 is associated with a broad range of pathologic conditions, including malignancies and various non-neoplastic diseases [11]. Studies suggest an miR-197induced regulating mechanism of PD-L1 in tumor cells via the miR-197/CKS1B/STAT3 pathway in non-small cell lung cancer (NSCLC) $[12,13]$. In those studies, downregulation of miR-197 was suggested as the promoter of chemoresistance, having therapeutic potential by miR-197 replacement, particularly in PD-L1-positive NSCLC patients.

In addition to miR-197, PD-L1 is regulated by other microRNAs, including miR-138-5p, miR-513, miR-570, miR-34a, and miR-200, the functions of which have not yet been elucidated in OSCC [14-18]. Considering previously reported clinically relevant signaling pathways involving CKS1B and STAT3 in OSCC and the known miR-197/CKS1B/STAT3/PD-L1 pathway [12, 13, 19-21], we focused on the miR-197/PD-L1 axis and TILs.

In the present study, we contribute to a better understanding of the clinicopathologic implication of the miR-197/PD-L1 axis and TILs in OSCC. We analyzed the expression of miR-197 and PD-L1 and analyzed the number of recruited TILs (CD3+, CD4+, CD8+, PD-1+, FoxP3+, and CD20+ lymphocytes) and the correlations with various clinicopathologic features and prognosis in OSCC patients.

\section{RESULTS}

\section{Clinicopathologic characteristics of OSCC patients}

The various clinicopathologic features of OSCC patients are summarized in Table 1. They were from 45 male patients and 23 female patients, with a median age of 57.7 years (range $=23-84$ years). The distribution according to pathologic TNM (pTNM) staging classification by the seventh edition of the AJCC was as follows: 18 patients $(26.5 \%)$ were I; 17 patients $(25.0 \%)$ were II; 9 patients $(13.2 \%)$ were III, and 24 patients $(35.3 \%)$ were IV. Among the 68 patients, grading categories of immunohistochemical intensity of PD-L1 expression in tumor cells were distributed as no staining (0; 23/68, 33.8\%), weak positivity $(1+; 23 / 68,33.8 \%)$, and moderate to strong positivity $(2+; 22 / 68,32.4 \%)$. The numbers of $\mathrm{CD} 3+, \mathrm{CD} 4+, \mathrm{CD} 8+, \mathrm{PD}-1+$, FoxP3+, and CD20+ TILs are shown in Table 1. PD-1+ TILs and FoxP3+ TILs were less dominant, compared with CD3+, CD4+, CD8+, and CD20+ TILs.

\section{Associations among clinicopathologic features and miR-197, PD-L1, and TILs in OSCC patients}

Clinicopathologic features were not significantly different between miR-197 $7^{\text {low }}\left(<\right.$ median) and miR-197 $7^{\text {high }}$ $(>$ median) subgroups or between low PD-L1 $(0 / 1+)$ and high PD-L1 (2+) subgroups (Supplementary Tables 1 and 2). In the quantitative analysis of miR-197 and TILs according to clinicopathologic features (Table 2), miR-197 level was higher in male patients $(P=0.049)$, and PD-1+ TILs were increased in older patients $(P=$ 0.018). The parameters of tumor aggressiveness were associated with depletion of various TILs. Decreased PD-1+, FoxP3+, and CD20+ TILs were associated with higher AJCC stages (3-4) $(P=0.010, P=0.004$, and $P$ $=0.035$, respectively). Specifically, high $\mathrm{T}$ stage (T3T4) was correlated with low numbers of CD3+, $\mathrm{CD} 8+$, PD-1+, FoxP3+, and CD20+ TILs $(P=0.019, P=0.030$, $P=0.019, P=0.005$, and $P=0.004$, respectively), and high N stage (N1-N2) disease had fewer PD-1+ TILs in the primary tumor $(P=0.020)$. In addition, the tumors with angiolymphatic invasion had fewer CD20+ TILs $(P=0.006)$, and perineural invasion was associated with fewer PD-1+ and FoxP3+ TILs $(P=0.022$ and $P=0.017)$. CD4+ TIL numbers were lower in patients who died $(P=0.035)$. When $\mathrm{T}$ stage was divided into T1-T3 versus T4, T4 disease had a higher miR-197 level $(P=0.049$; Figure 1 and Table 3$)$ but low PDL1 expression $(P=0.002$; Table 3$)$. Taken together, aggressive features were associated with higher miR-197 level, but depleted TILs and low PD-L1 expression.

\section{Associations between the miR-197/PD-L1 axis and microenvironmental TILs}

Based on research that suggests miR-197 indirectly suppresses PD-L1 expression [12], we analyzed the relation between level of miR-197 and PD-L1. As shown in Figure 1, Figure 2 and Table 3, miR-197 expression is inversely correlated with PD-L1 expression $(r=-0.239$, $P=0.050$, for $0,1+$, and $2+$ of PD-L1 intensity; $r=-0.293$, $P=0.015$, for 0 vs. $1+/ 2+$ of PD-L1 intensity). Because PD-L1 is associated with proliferation and function of TILs, we comparatively analyzed the relations among CD3+, CD4+, CD8+, PD-1+, FoxP3+, CD20+ TILs, and PD-L1 expression (Table 3). As shown in Figure 1 and 
Table 1: Clinicopathologic features of oral squamous cell carcinoma $(n=68)$

\begin{tabular}{|c|c|}
\hline Variables & \\
\hline \multicolumn{2}{|l|}{ Age, y } \\
\hline mean (range) & $57.7(23-84)$ \\
\hline \multicolumn{2}{|l|}{ Sex } \\
\hline $\mathrm{F}$ & $23(33.8 \%)$ \\
\hline M & $45(66.2 \%)$ \\
\hline \multicolumn{2}{|l|}{ Angiolymphatic invasion } \\
\hline Absent & $49(72.1 \%)$ \\
\hline Present & $19(27.9 \%)$ \\
\hline \multicolumn{2}{|l|}{ Perineural invasion } \\
\hline Absent & $49(72.1 \%)$ \\
\hline Present & $19(27.9 \%)$ \\
\hline Tumor size (largest dimension, mm) & $28.9(12-85)$ \\
\hline \multicolumn{2}{|l|}{$\mathrm{pT}$} \\
\hline pT1 & $21(30.9 \%)$ \\
\hline pT2 & $26(38.2 \%)$ \\
\hline pT3 & $4(5.9 \%)$ \\
\hline pT4a & $17(25.0 \%)$ \\
\hline \multicolumn{2}{|l|}{$\mathrm{pN}$} \\
\hline pN0 & $42(61.8 \%)$ \\
\hline $\mathrm{pN} 1$ & $15(22.1 \%)$ \\
\hline $\mathrm{pN} 2 \mathrm{~b}$ & $10(14.7 \%)$ \\
\hline $\mathrm{pN} 2 \mathrm{c}$ & $1(1.5 \%)$ \\
\hline \multicolumn{2}{|l|}{ pTNM } \\
\hline I & $18(26.5 \%)$ \\
\hline II & $17(25.0 \%)$ \\
\hline III & $9(13.2 \%)$ \\
\hline IV & $24(35.3 \%)$ \\
\hline \multicolumn{2}{|l|}{ Number of TILs } \\
\hline $\mathrm{CD} 3+$ TILs, mean $\pm \mathrm{SD}$ & $42.5 \pm 37.0$ \\
\hline $\mathrm{CD} 4+\mathrm{TILs}$, mean $\pm \mathrm{SD}$ & $32.6 \pm 26.7$ \\
\hline $\mathrm{CD} 8+\mathrm{TILs}$, mean $\pm \mathrm{SD}$ & $27.5 \pm 22.2$ \\
\hline PD-1+ TILs, mean \pm SD & $6.8 \pm 6.9$ \\
\hline FoxP3+ TILs, mean \pm SD & $9.4 \pm 9.4$ \\
\hline $\mathrm{CD} 20+\mathrm{TILs}$, mean $\pm \mathrm{SD}$ & $21.7 \pm 23.2$ \\
\hline \multicolumn{2}{|l|}{ PD-L1 expression } \\
\hline 0 & $23(33.8 \%)$ \\
\hline
\end{tabular}

(Continued) 


\begin{tabular}{lc}
\hline Variables & \\
\hline $1+$ & $23(33.8 \%)$ \\
$2+$ & $22(32.4 \%)$ \\
Survival & $45(66.2 \%)$ \\
Alive & $23(33.8 \%)$ \\
Death & \\
Relapse & $49(72.1 \%)$ \\
No relapse & $19(27.9 \%)$ \\
Relapse & \\
Neoadjuvant treatment & $65(95.6 \%)$ \\
Not done & $3(4.4 \%)$ \\
Done & \\
Additional treatment* & \\
Not done & $31(45.6 \%)$ \\
RTx only & $26(38.2 \%)$ \\
CTx only & $2(3.0 \%)$ \\
CCRT or SCRT & $9(13.2 \%)$ \\
\hline
\end{tabular}

TILs, tumor-infiltrating lymphocytes; SD, standard deviation; CCRT, concurrent chemoradiotherapy; SCRT, sequential chemoradiotherapy; CTx, chemotherapy; RTx, radiotherapy.

* Neoadjuvant treatment ( 3 cases) includes one neoadjuvant CCRT, one neoadjuvant RTx, and one neoadjuvant CTx.

**Additional treatment (37 cases) includes neoadjuvant treatment $(3 / 37,8 \%)$ and postoperative therapy $(34 / 37,92 \%)$.

Table 3, PD-L1 expression in tumor cells was positively correlated with the numbers of CD3+, CD4+, CD8+, and FoxP3 TILs $(P=0.027, P=0.037, P=0.003$, and $P=$ 0.006 , respectively), but not with PD-1+ TILs or CD20+ TILs $(P>0.05)$. When the relation among various TILs was analyzed, frequent positive correlations were observed among CD3+, CD4+, CD8+, FoxP3+, and CD20+ TILs.

\section{The effects of miR-197 and PD-L1 expression and TILs on prognosis in OSCC}

The association between conventional clinicopathologic factors and prognosis in OSCC patients was evaluated (Table 4 and Figure 3). Univariate analysis revealed that T stage (T3-T4; $P<0.001)$ and angiolymphatic invasion $(P=0.001)$ were predictors of poor OS in OSCC patients. Similarly, T stage (T3-T4; $P<0.001)$, angiolymphatic invasion $(P=0.001)$, and $\mathrm{N}$ stage $(P=0.007)$ were associated with poor DFS. High expression of miR-197 was weakly associated with poor OS $(P=0.033)$, and high PD-L1 expression in tumor cells $(2+)$ predicted better OS $(P=0.039$; Figure 3$)$, as expected from the inverse correlation between miR-197 and PD-L1. In multivariate analysis, T stage (T3-T4) and angiolymphatic invasion were independent poor prognostic factors for OS $(P<0.001$ and $P=0.002)$, and
DFS $(P=0.001$ and $P=0.003)$, but miR-197 or PD-L1 was not associated with OS or DFS.

\section{Survival analysis in miR-197 $7^{\text {low }}$ and $\mathrm{miR}-197^{\text {high }}$ subgroups}

Because our results suggested that miR-197 inhibited PD-L1 expression in OSCC, we investigated correlations of PD-L1 with survival according to miR-197 level (Table 5 and Figure 3). In the miR-197 high subgroup (>median value of miR-197; $\mathrm{n}=34$ ), prognostic factors for OS in univariate analysis, namely, PD-L1, T stage (T3-T4), and angiolymphatic invasion, were incorporated into the multivariate analysis, resulting in PD-L1 and angiolymphatic invasion as independent prognostic factors for OS $(P=0.040$ and $P=0.035)$, whereas angiolymphatic invasion was the only prognostic factor for DFS $(P=$ $0.009)$. In contrast, multivariate analysis of the miR$197^{\text {low }}$ subgroup ( $<$ median value of miR-197; $\mathrm{n}=34$ ) showed that $\mathrm{T}$ stage (T3-T4) and angiolymphatic invasion were independently associated with OS $(P=0.005$ and $P=0.040)$, and T stage (T3-T4) was an independent prognostic factor for DFS $(P=0.001)$, with no prognostic significance of PD-L1 for OS or DFS in the miR-197 low subgroup. Therefore, high PD-L1 expression $(2+)$ was a favorable prognostic factor for OS only in the miR-197 $7^{\text {high }}$ 
Table 2: Average numbers of tumor-infiltrating lymphocytes and level of miR-197 according to clinicopathologic features of oral squamous cell carcinoma $(n=68)$

\begin{tabular}{|c|c|c|c|c|c|c|c|c|c|c|c|c|c|c|c|}
\hline \multirow{2}{*}{$\begin{array}{l}\text { Clinicopathologic } \\
\text { variables }\end{array}$} & \multirow{2}{*}{$\begin{array}{c}\text { Number } \\
\text { of } \\
\text { patients } \\
\text { (total } \\
\text { n=68) }\end{array}$} & \multicolumn{2}{|l|}{ CD3+ } & \multicolumn{2}{|l|}{ CD4+ } & \multicolumn{2}{|l|}{ CD8+ } & \multicolumn{2}{|l|}{ PD-1+ } & \multicolumn{2}{|l|}{ FoxP3+ } & \multirow{2}{*}{$\frac{\mathrm{CD20}+}{\mathrm{Mean} \pm \mathrm{SD}}$} & \multicolumn{3}{|c|}{ miR-197 } \\
\hline & & $\begin{array}{c}\text { Mean } \pm \\
\text { SD }\end{array}$ & $\begin{array}{c}P \text { - } \\
\text { value }\end{array}$ & $\begin{array}{l}\text { Mean } \pm \\
\text { SD }\end{array}$ & $\begin{array}{c}P \text { - } \\
\text { value }\end{array}$ & $\begin{array}{l}\text { Mean } \pm \\
\text { SD }\end{array}$ & $\begin{array}{c}P- \\
\text { value }\end{array}$ & $\begin{array}{l}\text { Mean } \pm \\
\text { SD }\end{array}$ & $\begin{array}{c}P \text { - } \\
\text { value }\end{array}$ & $\begin{array}{c}\text { Mean } \pm \\
\text { SD }\end{array}$ & $\begin{array}{c}P \text { - } \\
\text { value }\end{array}$ & & $\begin{array}{c}P \text { - } \\
\text { value }\end{array}$ & $\begin{array}{l}\text { Mean } \pm \\
\text { SD }\end{array}$ & $\begin{array}{c}P \text { - } \\
\text { value }\end{array}$ \\
\hline Age & & & 0.579 & & 0.709 & & 0.725 & & 0.018 & & 0.395 & & 0.569 & & 0.282 \\
\hline$<55$ & 25 & $39.1 \pm 40.8$ & & $31.0 \pm 24.6$ & & $26.2 \pm 20.7$ & & $4.6 \pm 3.8$ & & $8.1 \pm 7.5$ & & $19.6 \pm 22.6$ & & $5.4 \pm 9.9$ & \\
\hline$\geq 55$ & 43 & $44.6 \pm 35.0$ & & $33.5 \pm 28.1$ & & $28.2 \pm 23.3$ & & $8.1 \pm 7.9$ & & $10.2 \pm 10.4$ & & $23.0 \pm 23.8$ & & $12.4 \pm 31.2$ & \\
\hline Gender & & & 0.512 & & 0.494 & & 0.434 & & 0.706 & & 0.175 & & 0.466 & & 0.049 \\
\hline Male & 45 & $40.3 \pm 33.4$ & & $34.2 \pm 28.4$ & & $26.0 \pm 20.8$ & & $7.0 \pm 7.0$ & & $10.4 \pm 10.8$ & & $23.2 \pm 23.5$ & & $13.0 \pm 31.1$ & \\
\hline Female & 23 & $46.7 \pm 43.6$ & & $29.5 \pm 23.3$ & & $30.5 \pm 25.0$ & & $6.4 \pm 6.8$ & & $7.6 \pm 5.9$ & & $18.8 \pm 22.9$ & & $3.6 \pm 2.3$ & \\
\hline AJCC Stage & & & 0.057 & & 0.145 & & 0.116 & & 0.010 & & 0.004 & & 0.035 & & 0.538 \\
\hline Stage 1-2 & 35 & $50.7 \pm 35.0$ & & $37.2 \pm 30.9$ & & $31.6 \pm 24.2$ & & $8.8 \pm 8.4$ & & $12.5 \pm 10.2$ & & $27.4 \pm 23.3$ & & $8.0 \pm 14.6$ & \\
\hline Stage 3-4 & 33 & $33.5 \pm 37.6$ & & $27.7 \pm 20.7$ & & $23.1 \pm 19.3$ & & $4.6 \pm 3.8$ & & $6.0 \pm 7.3$ & & $15.5 \pm 21.9$ & & $11.8 \pm 33.8$ & \\
\hline $\begin{array}{l}\text { AJCC Tumor } \\
\text { Stage }\end{array}$ & & & 0.019 & & 0.097 & & 0.030 & & 0.019 & & 0.005 & & 0.004 & & 0.280 \\
\hline pT1-2 & 47 & $49.6 \pm 39.2$ & & $36.2 \pm 28.7$ & & $30.9 \pm 23.9$ & & $7.8 \pm 7.8$ & & $11.4 \pm 9.5$ & & $26.1 \pm 25.6$ & & $6.6 \pm 12.8$ & \\
\hline pT3-4 & 21 & $26.9 \pm 26.3$ & & $24.6 \pm 19.7$ & & $19.9 \pm 16.0$ & & $4.5 \pm 3.5$ & & $5.0 \pm 7.7$ & & $12.1 \pm 12.7$ & & $17.0 \pm 41.9$ & \\
\hline $\begin{array}{l}\text { AJCC Lymph } \\
\text { node Stage }\end{array}$ & & & 0.454 & & 0.422 & & 0.739 & & 0.020 & & 0.128 & & 0.343 & & 0.982 \\
\hline $\mathrm{pN} 0$ & 42 & $45.1 \pm 34.7$ & & $34.7 \pm 29.5$ & & $28.2 \pm 23.7$ & & $8.1 \pm 8.0$ & & $10.8 \pm 10.1$ & & $23.8 \pm 22.8$ & & $9.9 \pm 20.3$ & \\
\hline $\mathrm{pN} 1-2$ & 26 & $38.1 \pm 40.9$ & & $29.3 \pm 21.4$ & & $26.4 \pm 20.0$ & & $4.7 \pm 3.8$ & & $7.1 \pm 8.0$ & & $18.2 \pm 24.1$ & & $9.8 \pm 33.0$ & \\
\hline $\begin{array}{l}\text { Angiolymphatic } \\
\text { invasion }\end{array}$ & & & 0.286 & & 0.437 & & 0.163 & & 0.197 & & 0.465 & & 0.006 & & 0.336 \\
\hline Not identified & 49 & $45.5 \pm 39.3$ & & $32.0 \pm 27.3$ & & $27.9 \pm 22.3$ & & $8.0 \pm 7.5$ & & $9.9 \pm 9.2$ & & $25.3 \pm 25.3$ & & $8.3 \pm 18.8$ & \\
\hline Present & 19 & $34.5 \pm 29.5$ & & $34.1 \pm 25.6$ & & $26.5 \pm 22.8$ & & $3.7 \pm 3.7$ & & $8.0 \pm 10.2$ & & $12.1 \pm 12.3$ & & $13.7 \pm 38.6$ & \\
\hline $\begin{array}{l}\text { Perineural } \\
\text { invasion }\end{array}$ & & & 0.947 & & 0.783 & & 0.815 & & 0.022 & & 0.017 & & 0.455 & & 0.439 \\
\hline Not identified & 49 & $42.3 \pm 30.7$ & & $32.0 \pm 27.3$ & & $27.9 \pm 22.3$ & & $8.0 \pm 7.5$ & & $10.8 \pm 10.2$ & & $23.1 \pm 22.0$ & & $8.3 \pm 18.8$ & \\
\hline Present & 19 & $43.1 \pm 50.1$ & & $34.1 \pm 25.6$ & & $26.5 \pm 22.8$ & & $3.7 \pm 3.7$ & & $5.8 \pm 6.2$ & & $18.3 \pm 26.5$ & & $13.7 \pm 38.6$ & \\
\hline Survival & & & 0.144 & & 0.035 & & 0.137 & & 0.459 & & 0.398 & & & & 0.123 \\
\hline Alive & 45 & $47.3 \pm 40.7$ & & $37.4 \pm 29.2$ & & $30.4 \pm 21.7$ & & $6.3 \pm 5.4$ & & $10.1 \pm 9.4$ & & $24.6 \pm 25.7$ & & $5.1 \pm 8.2$ & \\
\hline Death & 23 & $33.4 \pm 27.1$ & & $23.1 \pm 17.9$ & & $21.9 \pm 22.6$ & & $7.8 \pm 9.2$ & & $8.0 \pm 9.6$ & & $16.3 \pm 16.70 .170$ & & $19.1 \pm 41.6$ & \\
\hline Relapse & & & 0.923 & & 0.327 & & 0.833 & & 0.612 & & 0.511 & & 0.491 & & 0.333 \\
\hline No relapse & 49 & $42.2 \pm 39.2$ & & $34.6 \pm 27.7$ & & $27.9 \pm 22.0$ & & $7.1 \pm 6.7$ & & $8.8 \pm 8.3$ & & $23.0 \pm 25.3$ & & $7.2 \pm 16.4$ & \\
\hline Relapse & 19 & $43.2 \pm 31.9$ & & $27.5 \pm 23.8$ & & $26.6 \pm 23.4$ & & $6.1 \pm 7.5$ & & $10.8 \pm 12.1$ & & $18.6 \pm 17.0$ & & $16.7 \pm 40.8$ & \\
\hline $\begin{array}{l}\text { Neoadjuvant } \\
\text { treatment }\end{array}$ & & & 0.109 & & 0.557 & & 0.142 & & 0.477 & & 0.232 & & 0.155 & & 0.751 \\
\hline Not done & 65 & $44.1 \pm 37.1$ & & $33.0 \pm 27.0$ & & $28.4 \pm 22.4$ & & $6.9 \pm 7.0$ & & $9.7 \pm 9.6$ & & $22.6 \pm 23.4$ & & $10.0 \pm 26.2$ & \\
\hline Done & 3 & $9.0 \pm 5.3$ & & $23.7 \pm 20.4$ & & $9.0 \pm 6.6$ & & $4.0 \pm 3.6$ & & $3.0 \pm 2.0$ & & $3.0 \pm 2.6$ & & $5.2 \pm 5.2$ & \\
\hline $\begin{array}{l}\text { Additional } \\
\text { treatment }\end{array}$ & & & 0.978 & & 0.981 & & 0.996 & & 0.303 & & 0.051 & & 0.634 & & 0.132 \\
\hline Not done & 31 & $42.4 \pm 34.7$ & & $32.5 \pm 28.2$ & & $27.5 \pm 22.6$ & & $7.7 \pm 7.6$ & & $11.9 \pm 10.3$ & & $23.2 \pm 24.1$ & & $5.0 \pm 8.7$ & \\
\hline Done & 37 & $42.6 \pm 39.3$ & & $32.7 \pm 25.7$ & & $27.5 \pm 22.3$ & & $6.0 \pm 6.2$ & & $7.4 \pm 8.3$ & & $20.5 \pm 22.8$ & & $13.8 \pm 33.5$ & \\
\hline
\end{tabular}


Table 3: Correlations among tumor PD-L1 expression, miR-197 level, tumor-infiltrating lymphocytes, and pathologic stages in oral squamous cell carcinoma $(n=68)$

\begin{tabular}{|c|c|c|c|c|c|c|c|c|c|}
\hline & $\begin{array}{c}\text { PD-L1 } \\
\text { expression } \\
\text { (0 vs. } \\
1+/ 2+)\end{array}$ & $\begin{array}{c}\text { CD3+ } \\
\text { TIL } \\
\text { number }\end{array}$ & $\begin{array}{c}\text { CD4+ } \\
\text { TIL } \\
\text { number }\end{array}$ & $\begin{array}{c}\text { CD8+ } \\
\text { TIL } \\
\text { number }\end{array}$ & $\begin{array}{c}\text { PD-1+ } \\
\text { TIL } \\
\text { number }\end{array}$ & $\begin{array}{c}\text { FoxP3+ } \\
\text { TIL } \\
\text { number }\end{array}$ & $\begin{array}{c}\text { CD20+ } \\
\text { TIL } \\
\text { number }\end{array}$ & $\begin{array}{c}\text { T stage } \\
\text { (T1-3 vs. } \\
\text { T4) }\end{array}$ & $\begin{array}{c}\text { N stage } \\
\text { (N0 vs. } \\
\text { N1-2) }\end{array}$ \\
\hline miR-197 & $P=0.015$ & $P=0.316$ & $P=0.291$ & $P=0.291$ & $P=0.787$ & $P=0.307$ & $P=0.207$ & $P=0.049$ & $P=0.982$ \\
\hline expression & $\mathrm{r}=-0.293$ & $\mathrm{r}=-0.124$ & $\mathrm{r}=-0.130$ & $\mathrm{r}=-0.130$ & $\mathrm{r}=-0.033$ & $\mathrm{r}=-0.127$ & $\mathrm{r}=-0.156$ & $\mathrm{r}=0.239$ & $\mathrm{r}=-0.003$ \\
\hline PD-L1 & & $P=0.027$ & $P=\mathbf{0 . 0 3 7}$ & $P=0.003$ & $P=0.295$ & $P=0.006$ & $P=0.138$ & $P=0.002 *$ & $P=0.648 *$ \\
\hline $\begin{array}{l}\text { expression } \\
(0,1+, 2+)\end{array}$ & & $\mathrm{r}=0.271$ & $\mathrm{r}=0.254$ & $\mathrm{r}=0.352$ & $\mathrm{r}=0.129$ & $\mathrm{r}=0.333$ & $\mathrm{r}=0.183$ & $\mathrm{r}=-0.371^{*}$ & $\mathrm{r}=-0.057 *$ \\
\hline CD3+ TIL & & & $P<0.001$ & $P<0.001$ & $P=0.169$ & $P<0.001$ & $P<0.001$ & $P=0.047$ & $P=0.454$ \\
\hline number & & & $\mathrm{r}=0.585$ & $r=0.659$ & $\mathrm{r}=0.170$ & $\mathrm{r}=0.440$ & $\mathrm{r}=0.741$ & $\mathrm{r}=0.243$ & $\mathrm{r}=-0.093$ \\
\hline CD4+ TIL & & & & $P<0.001$ & $P=0.737$ & $P=0.001$ & $P<0.001$ & $P=0.225$ & $P=0.422$ \\
\hline number & & & & $r=0.679$ & $\mathrm{r}=0.041$ & $\mathrm{r}=0.386$ & $\mathrm{r}=0.420$ & $\mathrm{r}=-0.149$ & $\mathrm{r}=-0.099$ \\
\hline CD8+ TIL & & & & & $P=0.155$ & $P<0.001$ & $P<0.001$ & $P=0.061$ & $P=0.739$ \\
\hline number & & & & & $\mathrm{r}=0.174$ & $\mathrm{r}=0.471$ & $\mathrm{r}=0.419$ & $\mathrm{r}=-0.228$ & $\mathrm{r}=-0.041$ \\
\hline PD-1+ TIL & & & & & & $P=0.055$ & $P=0.115$ & $P=0.118$ & $P=0.020$ \\
\hline number & & & & & & $\mathrm{r}=0.235$ & $\mathrm{r}=0.194$ & $\mathrm{r}=-0.191$ & $\mathrm{r}=-0.246$ \\
\hline FoxP3+ & & & & & & & $P=0.017$ & $P<0.001$ & $P=0.128$ \\
\hline $\begin{array}{l}\text { TIL } \\
\text { number }\end{array}$ & & & & & & & $\begin{array}{c}r= \\
-0.292\end{array}$ & $\mathrm{r}=-0.417$ & $\mathrm{r}=-0.188$ \\
\hline CD20+ & & & & & & & & $P=0.029$ & $P=0.343$ \\
\hline number & & & & & & & & $r=-0.266$ & $\mathrm{r}=-0.118$ \\
\hline T stage & & & & & & & & & $P=0.044^{*}$ \\
\hline (T1-3 vs. & & & & & & & & & $\mathrm{r}=0.245^{*}$ \\
\hline & & & & & & & & & \\
\hline
\end{tabular}

TIL, tumor-infiltrating lymphocyte. $P$ and $\mathrm{r}$ indicate $P$-value and correlation coefficient from Pearson correlation test. Asterisk (*) indicates $P$-value and correlation coefficient from Spearman's rho test.

subgroup, suggesting that prognostic implication of PD-L1 is influenced by the miR-197 level.

\section{DISCUSSION}

In the tumor microenvironment, the PD-L1/ PD-1 axis has become the therapeutic locus in various cancers. In this study, we focused on the clinicopathologic implication of the miR-197/PD-L1 axis and the profiles of recruited TILs in OSCC. We observed the inverse correlation between miR-197 and PD-L1, and the positive correlation between PD-L1 and TILS, suggesting an active network of miR-197/PD-L1/TILs. Aggressive clinicopathologic features were associated with depleted TILs, and high T stage (T4) disease was associated with low PD-L1 but high miR-197 expression. Moreover, high PD-L1 expression (2+) was an independent favorable prognostic factor for overall survival (OS) $(P=0.040)$ in the miR-197 $7^{\text {high }}$ subgroup, but not in the miR-197 $7^{\text {low }}$ subgroup.
MiR-197 is upregulated in lung, liver, and thyroid cancers and targets a variety of genes promoting cell proliferation and inhibiting apoptosis $[11,22,23]$. MiR197 has an indirect effect on PD-L1, which has already been investigated in a study of NSCLC [12]. In the NSCLC study, the miR-197/CKS1B/STAT3-induced PD-L1 network leads to tumor progression, and miR-197 is an inverse indicator of PD-L1 expression and predicts shorter OS [12]. Consistent with this result, we observed that miR-197 level inversely correlated with PD-L1 expression detected by immunohistochemistry in OSCC, suggesting that miR-197, at least in part, suppresses PD-L1 expression in OSCC. The proposed mechanism in NSCLC explains that miR-197 has a direct inhibitory effect on the cyclin-dependent kinase CKS1B, which promotes PD-L1 expression by activating STAT3 [12]. Although the detailed mechanism of PD-L1 expression remains to be clarified, our observations suggest that the putative miR197-mediated negative regulation of PD-L1 might also have clinical significance in OSCC. 
The mechanisms of PD-L1 expression in tumor cells have been suggested to include two major pathways. The extrinsic signaling pathway is activated by interferon- $\gamma$ (IFN- $\gamma$ ) produced by TILs, resulting in JAK/STAT activation in tumor cells, and the intrinsic pathway is activated by $\mathrm{PI} 3 \mathrm{~K} / \mathrm{AKT} / \mathrm{mTOR}$ signaling $[24,25]$. In this context, miR-197 possibly suppress the downstream signaling of the extrinsic pathway of PD-L1 expression via CKS1B/STAT3 pathway, and therefore, the resultant PD-L1 expression level might reflect the signals from IFN- $\gamma$-producing T cells modified by miR-197, as well as signals from the intrinsic pathway. Considering the inverse correlation of miR-197 and PD-L1 in our study, PD-L1 expression in OSCC might be dependent on the miR-197-
A

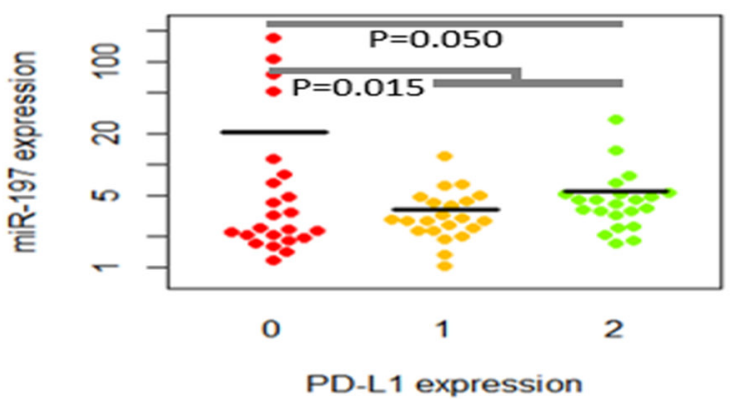

C

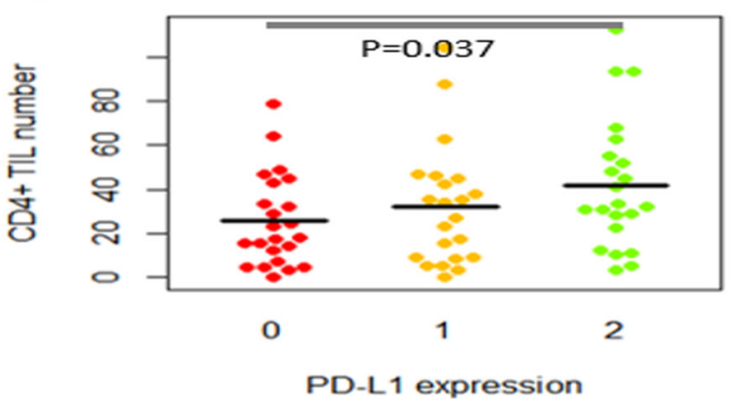

$\mathbf{E}$

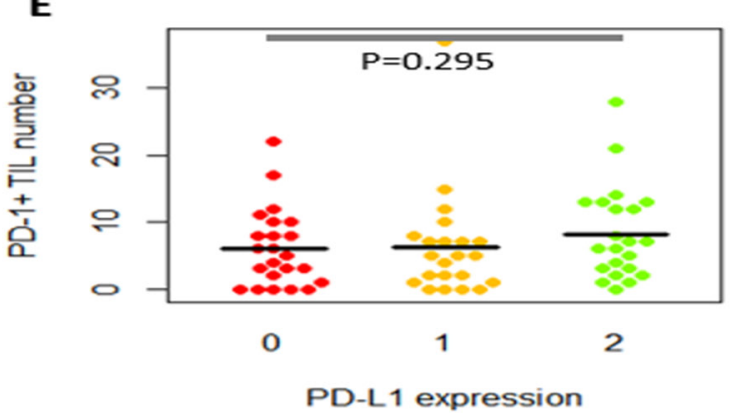

$\mathbf{G}$

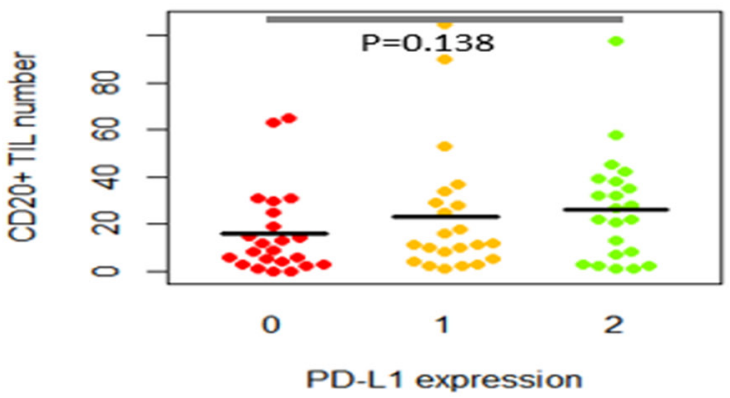

B

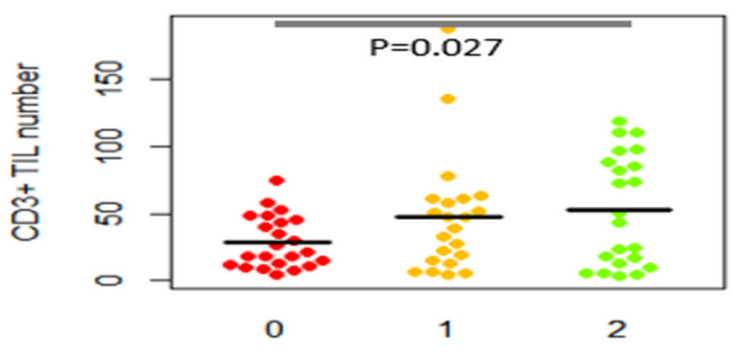

PD-L1 expression

D

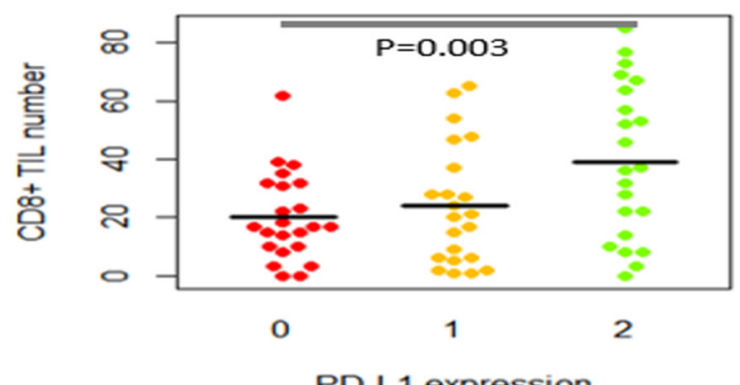

$\mathbf{F}$

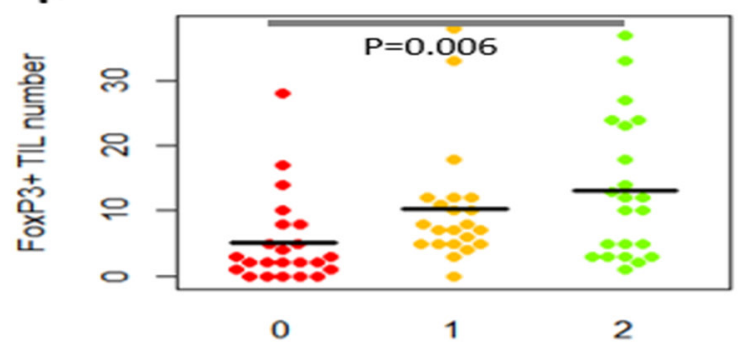

PD-L1 expression

$\mathbf{H}$

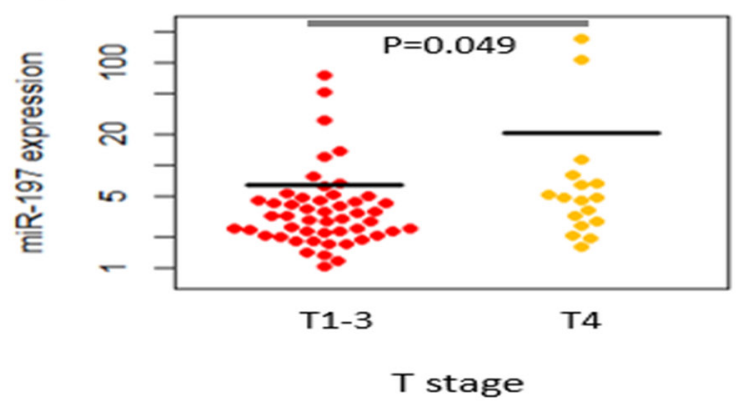

Figure 1: Dot plots of relative miR-197 expression level and tumor-infiltrating lymphocytes (TILs) according to PD-L1 expression and T stages. MiR-197 expression level (A) and the numbers of CD3+ (B), CD4+ (C), CD8+ (D), PD-1+ (E), FoxP3+ (F), and CD20+ (G) tumor-infiltrating lymphocytes (TILs) were illustrated according to PD-L1 level. PD-L1 expression is inversely correlated with miR-197 level (A) but tended to have a positive correlation with TILs (B-G). MiR-197 level is higher in T4 disease than in T1-T3 disease (H). 
mediated negative regulation, which could provide the different prognostic significance of PD-L1 according to the miR-197 level.

We divided our OSCC cohort into miR-197 high and miR-197 $7^{\text {low }}$ subsets, reflecting the mechanism of PDL1 expression. PD-L1 had prognostic significance only in the miR-197 ${ }^{\text {high }}$ subset, although it is not yet clear how high expression of miR-197 influences prognostic significance of PD-L1 in addition to expression of PD-L1. It is possible that OSCC with high miR-197 expression at a sufficient level might have an inhibitory effect on the signal from the extrinsic pathway induced
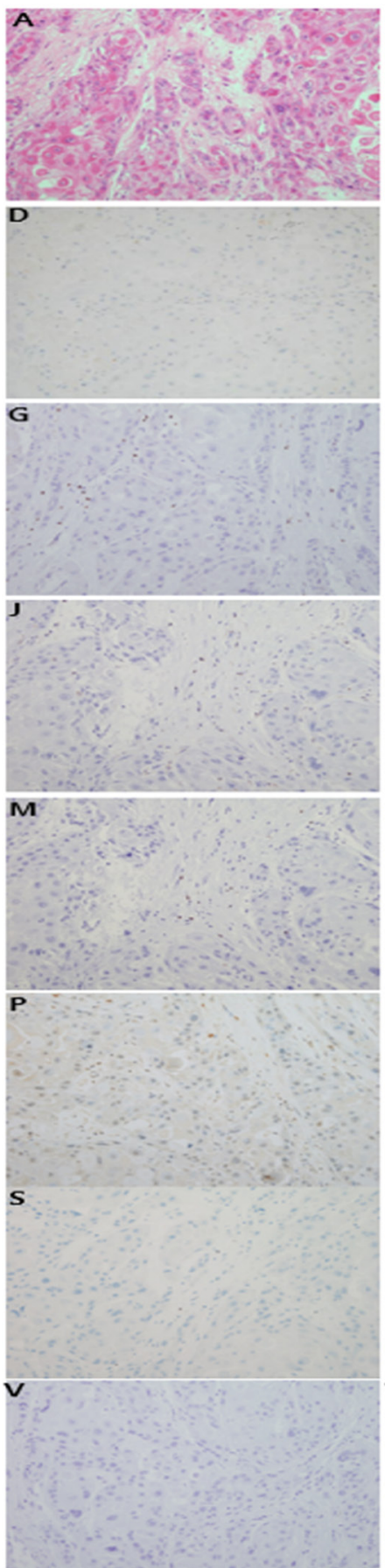
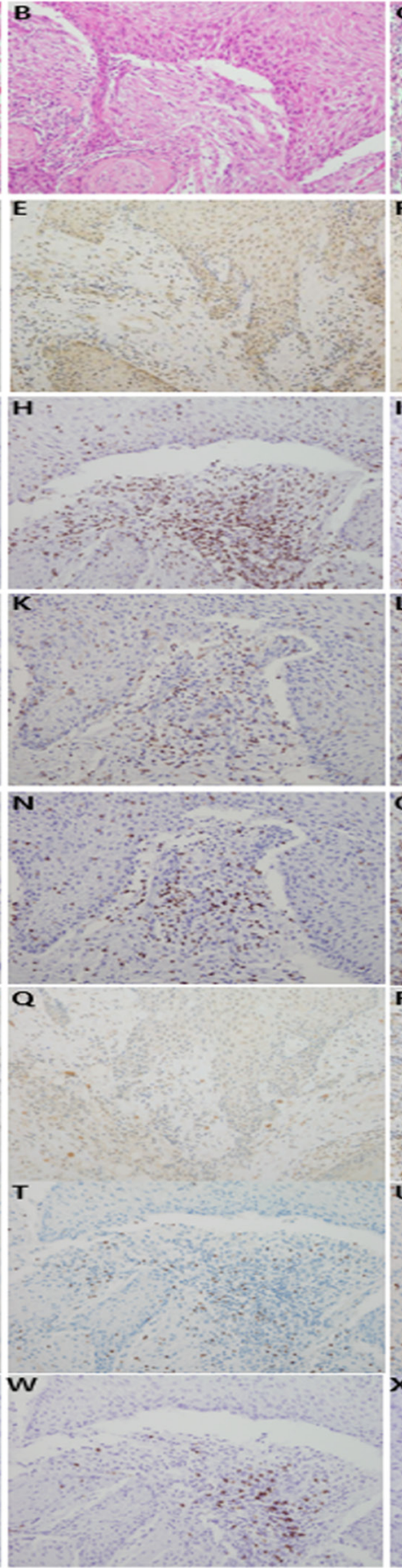
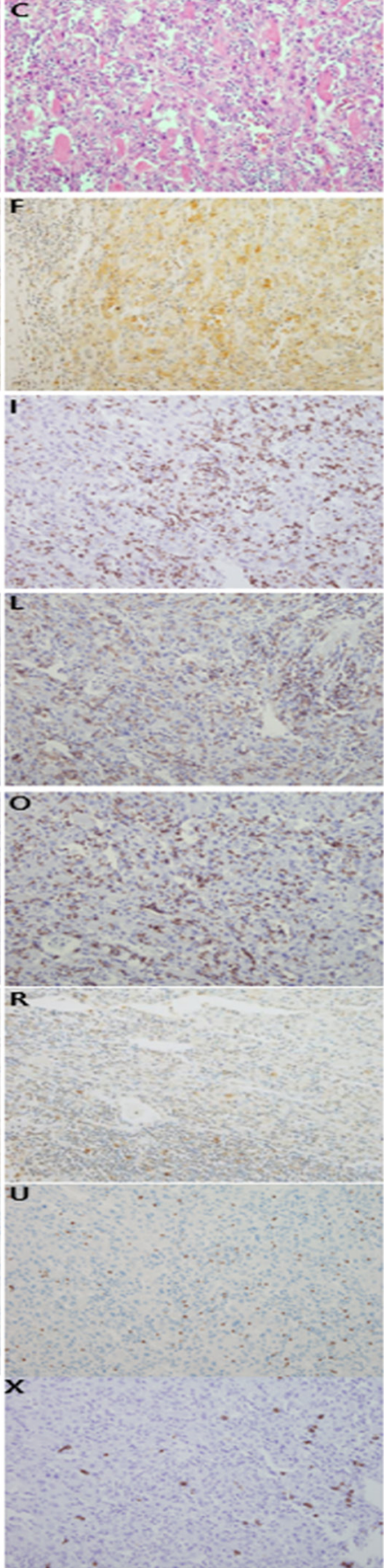

Figure 2: Representative features of histologic and immunohistochemical analyses. Histologic features of invasive oral squamous cell carcinomas are shown (hematoxylin and eosin stain, x200; A, B, C). Immunostaining of PD-L1 revealed no staining (0; D), weak positivity $(1+; \mathbf{E})$, and moderate to strong positivity $(2+; \mathbf{F})$. Tumor-infiltrating lymphocytes tended to be variably correlated with PD-L1 expression. The infiltrating lymphoid cells were immunostained with CD3 (G, H, I), CD4 (J, K, L), CD8 (M, N, O), PD-1 (P, Q, $\mathbf{R})$, FoxP3 (S, T, U), and CD20 (V, W, X). 
Table 4: Prognostic factors for overall survival and disease-free survival in oral squamous cell carcinoma $(\mathrm{n}=68)$

\begin{tabular}{|c|c|c|c|c|}
\hline \multirow[t]{2}{*}{ Variables } & \multicolumn{2}{|c|}{ Univariate analysis } & \multicolumn{2}{|c|}{ Multivariate analysis } \\
\hline & HR $(95 \%$ CI $)$ & $P$-value & HR (95\% CI) & $P$-value \\
\hline \multicolumn{5}{|l|}{ Overall survival } \\
\hline T Stage (T3-T4) & $5.68(2.44-13.22)$ & $<0.001$ & $5.21(2.20-12.37)$ & $<0.001$ \\
\hline $\begin{array}{l}\text { Angiolymphatic invasion } \\
\text { (present) }\end{array}$ & $4.21(1.85-9.61)$ & 0.001 & $3.72(1.60-8.65)$ & 0.002 \\
\hline $\mathrm{N}$ stage (N2) & $2.37(0.93-6.02)$ & 0.070 & & \\
\hline Age $(\geq 55)$ & $1.86(0.73-4.73)$ & 0.194 & & \\
\hline Gender (female) & $1.61(0.69-3.75)$ & 0.270 & & \\
\hline Perineural invasion (present) & $1.55(0.65-3.66)$ & 0.321 & & \\
\hline $\begin{array}{l}\text { PD-1+ cell number (high; } \\
\text { continuous variable) }\end{array}$ & $1.01(0.95-1.06))$ & 0.826 & & \\
\hline $\begin{array}{l}\text { miR-197 expression (high; } \\
\text { continuous variable) }\end{array}$ & $1.01(1.00-1.02)$ & 0.033 & & NS \\
\hline $\begin{array}{l}\text { CD8+ cell number (high; } \\
\text { continuous variable) }\end{array}$ & $0.99(0.97-1.01)$ & 0.181 & & \\
\hline $\begin{array}{l}\text { CD3+ cell number (high; } \\
\text { continuous variable) }\end{array}$ & $0.99(0.98-1.00)$ & 0.142 & & \\
\hline $\begin{array}{l}\text { CD4+ cell number (high; } \\
\text { continuous variable) }\end{array}$ & $0.98(0.96-1.00)$ & 0.082 & & \\
\hline $\begin{array}{l}\text { CD20+ cell number (high; } \\
\text { continuous variable) }\end{array}$ & $0.98(0.96-1.00)$ & 0.186 & & \\
\hline $\begin{array}{l}\text { FoxP3 + cell number (high; } \\
\text { continuous variable) }\end{array}$ & $0.98(0.93-1.03)$ & 0.374 & & \\
\hline PD-L1 expression (2+) & $0.32(0.11-0.94)$ & 0.039 & & NS \\
\hline \multicolumn{5}{|l|}{ Disease-free survival } \\
\hline T Stage (T3-T4) & $7.74(2.67-22.42)$ & $<0.001$ & $6.69(2.25-19.84)$ & 0.001 \\
\hline $\begin{array}{l}\text { Angiolymphatic invasion } \\
\text { (present) }\end{array}$ & $5.88(2.13-16.27)$ & 0.001 & $4.90(1.72-13.95)$ & 0.003 \\
\hline N stage (N2) & $4.04(1.46-11.15)$ & 0.007 & & NS \\
\hline Perineural invasion (present) & $2.35(0.87-6.31)$ & 0.092 & & \\
\hline Gender (female) & $1.68(0.63-4.52)$ & 0.303 & & \\
\hline $\begin{array}{l}\text { FoxP3 + cell number (high; } \\
\text { continuous variable) }\end{array}$ & $1.01(0.96-1.06)$ & 0.754 & & \\
\hline $\begin{array}{l}\text { miR-197 expression (high; } \\
\text { continuous variable) }\end{array}$ & $1.01(1.00-1.02)$ & 0.089 & & \\
\hline $\begin{array}{l}\text { CD3+ cell number (high; } \\
\text { continuous variable) }\end{array}$ & $1.00(0.98-1.01)$ & 0.552 & & \\
\hline $\begin{array}{l}\text { CD20+ cell number (high; } \\
\text { continuous variable) }\end{array}$ & $0.99(0.97-1.02)$ & 0.496 & & \\
\hline
\end{tabular}

(Continued) 


\begin{tabular}{|c|c|c|c|c|}
\hline \multirow[t]{2}{*}{ Variables } & \multicolumn{2}{|c|}{ Univariate analysis } & \multicolumn{2}{|c|}{ Multivariate analysis } \\
\hline & HR (95\% CI) & $P$-value & HR $(95 \%$ CI $)$ & $P$-value \\
\hline $\begin{array}{l}\text { CD8+ cell number (high; } \\
\text { continuous variable) }\end{array}$ & $0.99(0.96-1.01)$ & 0.282 & & \\
\hline $\begin{array}{l}\text { CD4+ cell number (high; } \\
\text { continuous variable) }\end{array}$ & $0.98(0.96-1.01)$ & 0.187 & & \\
\hline $\begin{array}{l}\text { PD-1+ cell number (high; } \\
\text { continuous variable) }\end{array}$ & $0.94(0.85-1.04))$ & 0.213 & & \\
\hline Age $(\geq 55)$ & $0.79(0.30-2.13)$ & 0.646 & & \\
\hline PD-L1 expression (2+) & $0.25(0.06-1.12)$ & 0.070 & & \\
\hline
\end{tabular}

HR, harzard ratio; CI, confidence interval; NS, not significant.

A

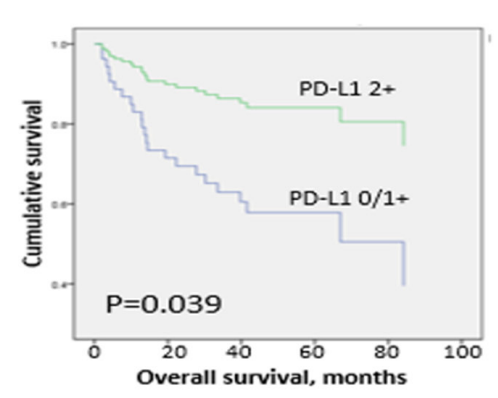

C

miR-197 high subgroup (>median) (OS)

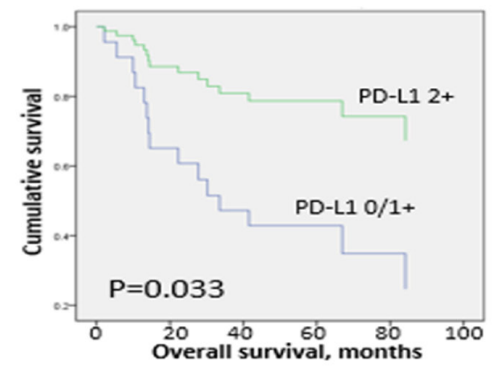

E

miR-197 low subgroup (<median) (OS)

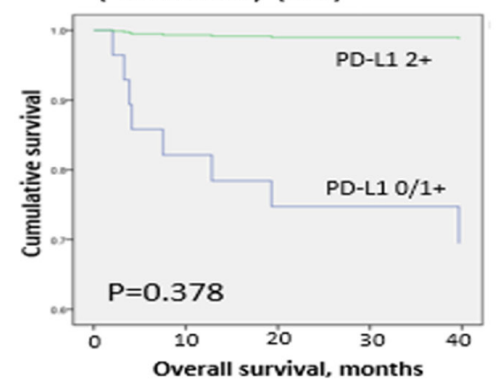

B

Full cohort (DFS)

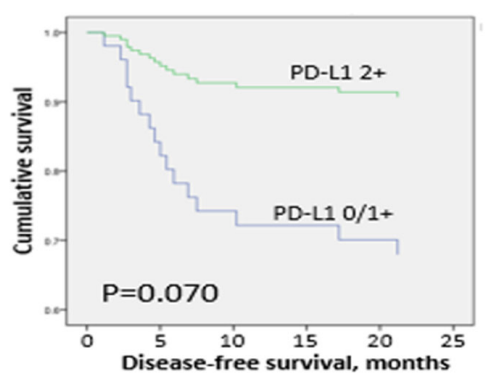

D

miR-197 high subgroup (>median) (DFS)

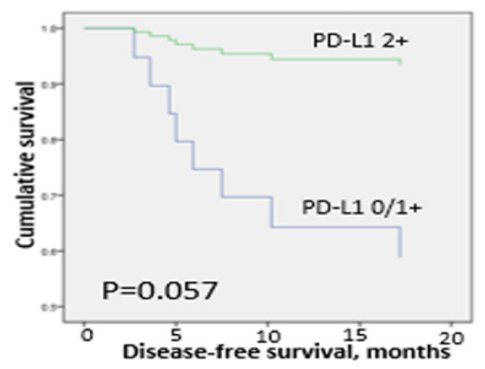

F

miR-197 low subgroup (<median) (DFS)

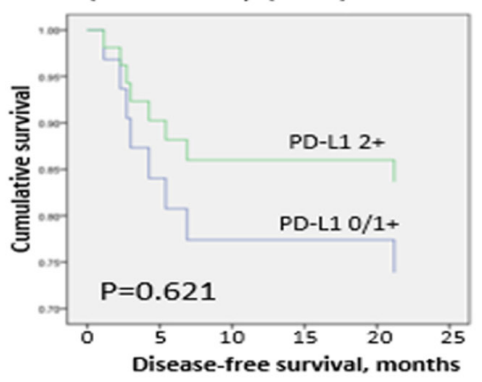

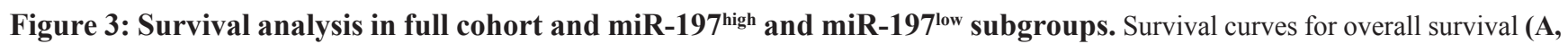
$\mathbf{C}, \mathbf{E})$ and disease-free survival (B, D, F) in full cohort (A and B), miR-197 $7^{\text {high }}(>$ median; C and $\mathbf{D})$ ), and miR-197 ${ }^{\text {low }}$ subgroups ( $<$ median; $\mathbf{E}$ and $\mathbf{F}$ ) in oral squamous cell carcinoma patients. 
Table 5: Prognostic factors for overall survival and disease-free survival in $\mathrm{miR}-197^{\mathrm{high}}(\mathrm{n}=34)$ and $\mathrm{miR}-197^{\mathrm{low}}$ $(n=34)$ subgroups of oral squamous cell carcinoma

\begin{tabular}{|c|c|c|c|c|}
\hline \multicolumn{5}{|c|}{ miR-197 ${ }^{\text {high }}$ subgroup (miR-197 > median) } \\
\hline \multirow[t]{2}{*}{ Variables } & \multicolumn{2}{|c|}{ Univariate analysis ${ }^{\dagger}$} & \multicolumn{2}{|c|}{ Multivariate analysis ${ }^{\dagger}$} \\
\hline & HR $(95 \%$ CI) & $P$-value & HR $(95 \%$ CI) & $P$-value \\
\hline \multicolumn{5}{|l|}{ Overall survival } \\
\hline $\begin{array}{l}\text { Angiolymphatic invasion } \\
\text { (present) }\end{array}$ & $3.31(1.16-9.45)$ & 0.025 & $3.16(1.09-9.17)$ & 0.035 \\
\hline $\mathrm{N}$ stage $(\mathrm{N} 2)$ & $3.17(0.86-11.69)$ & 0.084 & & \\
\hline T Stage (T3-T4) & $2.85(1.02-7.96)$ & 0.045 & & NS \\
\hline Age $(\geq 55)$ & $2.13(0.60-7.57)$ & 0.243 & & \\
\hline Gender (female) & $1.43(0.48-4.24)$ & 0.523 & & \\
\hline $\begin{array}{l}\text { FoxP3 + cell number (high; } \\
\text { continuous variable) }\end{array}$ & $1.00(0.95-1.05)$ & 0.897 & & \\
\hline $\begin{array}{l}\text { CD8+ cell number (high; } \\
\text { continuous variable) }\end{array}$ & $0.99(0.97-1.01)$ & 0.463 & & \\
\hline $\begin{array}{l}\text { CD3+ cell number (high; } \\
\text { continuous variable) }\end{array}$ & $0.99(0.98-1.01)$ & 0.244 & & \\
\hline $\begin{array}{l}\text { CD4+ cell number (high; } \\
\text { continuous variable) }\end{array}$ & $0.99(0.97-1.01)$ & 0.366 & & \\
\hline $\begin{array}{l}\text { CD20+ cell number (high; } \\
\text { continuous variable) }\end{array}$ & $0.98(0.95-1.01)$ & 0.244 & & \\
\hline $\begin{array}{l}\text { PD-1+ cell number (high; } \\
\text { continuous variable) }\end{array}$ & $0.98(0.90-1.06)$ & 0.599 & & \\
\hline Perineural invasion (present) & $0.64(0.18-2.25)$ & 0.482 & & \\
\hline PD-L1 expression $(2+)$ & $0.28(0.09-0.90)$ & $\mathbf{0 . 0 3 3}$ & $0.29(0.09-0.95)$ & 0.040 \\
\hline \multicolumn{5}{|l|}{ Disease-free survival } \\
\hline $\begin{array}{l}\text { Angiolymphatic invasion } \\
\text { (present) }\end{array}$ & $6.92(1.64-29.33)$ & 0.009 & $6.92(1.64-29.33)$ & 0.009 \\
\hline N stage (N2) & $6.07(1.42-25.86)$ & 0.015 & & NS \\
\hline T Stage (T3-T4) & $4.15(0.99-17.40)$ & 0.052 & & \\
\hline Perineural invasion (present) & $1.57(0.38-6.59)$ & 0.535 & & \\
\hline Gender (female) & $1.49(0.36-6.23)$ & 0.587 & & \\
\hline Age $(\geq 55)$ & $1.47(0.30-7.29)$ & 0.637 & & \\
\hline $\begin{array}{l}\text { FoxP3 + cell number (high; } \\
\text { continuous variable) }\end{array}$ & $1.03(0.96-1.10)$ & 0.412 & & \\
\hline $\begin{array}{l}\text { CD3+ cell number (high; } \\
\text { continuous variable) }\end{array}$ & $0.99(0.97-1.01)$ & 0.418 & & \\
\hline $\begin{array}{l}\text { CD8+ cell number (high; } \\
\text { continuous variable) }\end{array}$ & $0.98(0.95-1.02)$ & 0.307 & & \\
\hline $\begin{array}{l}\text { CD20+ cell number (high; } \\
\text { continuous variable) }\end{array}$ & $0.98(0.94-1.02)$ & 0.325 & & \\
\hline
\end{tabular}

(Continued) 
miR-197 ${ }^{\text {low }}$ subgroup $($ miR-197 $<$ median)

\begin{tabular}{|c|c|c|c|c|}
\hline \multirow[t]{2}{*}{ Variables } & \multicolumn{2}{|c|}{ Univariate analysis $^{\dagger}$} & \multicolumn{2}{|c|}{ Multivariate analysis $^{\dagger}$} \\
\hline & HR (95\% CI) & $P$-value & HR $(95 \%$ CI $)$ & $P$-value \\
\hline $\begin{array}{l}\text { CD4+ cell number (high; } \\
\text { continuous variable) }\end{array}$ & $0.98(0.94-1.01)$ & 0.221 & & \\
\hline $\begin{array}{l}\text { PD-1+ cell number (high; } \\
\text { continuous variable) }\end{array}$ & $0.96(0.85-1.08)$ & 0.503 & & \\
\hline PD-L1 expression $(2+)$ & $0.13(0.02-1.06)$ & 0.057 & & \\
\hline \multicolumn{5}{|l|}{ Overall survival } \\
\hline T Stage (T3-T4) & $15.90(3.13-80.85)$ & 0.001 & $11.80(2.13-65.44)$ & 0.005 \\
\hline $\begin{array}{l}\text { Angiolymphatic invasion } \\
\text { (present) }\end{array}$ & $9.25(1.85-46.33)$ & 0.007 & $6.21(1.09-35.47)$ & 0.040 \\
\hline Perineural invasion (present) & $5.64(1.33-23.95)$ & 0.019 & & NS \\
\hline N stage (N2) & $2.75(0.66-11.54)$ & 0.166 & & \\
\hline Gender (female) & $1.98(0.49-7.96)$ & 0.334 & & \\
\hline Age $(\geq 55)$ & $1.36(0.32-5.68)$ & 0.678 & & \\
\hline $\begin{array}{l}\text { PD-1+ cell number (high; } \\
\text { continuous variable) }\end{array}$ & $1.02(0.94-1.10)$ & 0.675 & & \\
\hline $\begin{array}{l}\text { CD20+ cell number (high; } \\
\text { continuous variable) }\end{array}$ & $0.98(0.94-1.02)$ & 0.382 & & \\
\hline $\begin{array}{l}\text { CD3+ cell number (high; } \\
\text { continuous variable) }\end{array}$ & $0.97(0.93-1.01)$ & 0.135 & & \\
\hline $\begin{array}{l}\text { CD4+ cell number (high; } \\
\text { continuous variable) }\end{array}$ & $0.97(0.93-1.01)$ & 0.129 & & \\
\hline $\begin{array}{l}\text { CD8+ cell number (high; } \\
\text { continuous variable) }\end{array}$ & $0.94(0.88-1.00)$ & 0.054 & & \\
\hline $\begin{array}{l}\text { FoxP3 + cell number (high; } \\
\text { continuous variable) }\end{array}$ & $0.85(0.70-1.03)$ & 0.101 & & \\
\hline PD-L1 expression $(2+)$ & $0.04(0.00-59.97)$ & 0.378 & & \\
\hline \multicolumn{5}{|l|}{ Disease-free survival } \\
\hline T Stage (T3-T4) & $15.29(2.99-78.18)$ & 0.001 & $15.29(2.99-78.18)$ & 0.001 \\
\hline $\begin{array}{l}\text { Angiolymphatic invasion } \\
\text { (present) }\end{array}$ & $4.88(1.16-20.55)$ & 0.031 & & NS \\
\hline Perineural invasion (present) & $3.15(0.78-12.76)$ & 0.107 & & \\
\hline N stage (N2) & $2.92(0.70-12.27)$ & 0.144 & & \\
\hline Gender (female) & $1.88(0.47-7.52)$ & 0.374 & & \\
\hline $\begin{array}{l}\text { CD3 + cell number (high; } \\
\text { continuous variable) }\end{array}$ & $1.00(0.98-1.03)$ & 0.800 & & \\
\hline $\begin{array}{l}\text { CD20+ cell number (high; } \\
\text { continuous variable) }\end{array}$ & $1.00(0.97-1.03)$ & 0.913 & & \\
\hline $\begin{array}{l}\text { CD4+ cell number (high; } \\
\text { continuous variable) }\end{array}$ & $0.99(0.96-1.02)$ & 0.540 & & \\
\hline
\end{tabular}

(Continued) 
miR-197 ${ }^{\text {low }}$ subgroup (miR-197 < median)

\begin{tabular}{|c|c|c|c|c|}
\hline \multirow[t]{2}{*}{ Variables } & \multicolumn{2}{|c|}{ Univariate analysis $^{\dagger}$} & \multicolumn{2}{|c|}{ Multivariate analysis $^{\dagger}$} \\
\hline & HR $(95 \%$ CI $)$ & $P$-value & HR $(95 \%$ CI $)$ & $P$-value \\
\hline $\begin{array}{l}\text { CD8+ cell number (high; } \\
\text { continuous variable) }\end{array}$ & $0.99(0.95-1.04)$ & 0.741 & & \\
\hline $\begin{array}{l}\text { FoxP3 + cell number (high; } \\
\text { continuous variable) }\end{array}$ & $0.98(0.87-1.09)$ & 0.660 & & \\
\hline $\begin{array}{l}\text { PD-1+ cell number (high; } \\
\text { continuous variable) }\end{array}$ & $0.84(0.64-1.10)$ & 0.197 & & \\
\hline Age $(\geq 55)$ & $0.47(0.11-1.98)$ & 0.304 & & \\
\hline PD-L1 expression (2+) & $0.59(0.07-4.79)$ & 0.621 & & \\
\hline
\end{tabular}

by STAT3, while sparing the intrinsic signal. This skewing effect of the PD-L1 pathway with co-activated $\mathrm{Akt} / \mathrm{mTOR}$-related downstream signals could influence the biology of OSCC in a complex manner, which remains to be clarified further.

Attempts have been made to identify the relation between PD-L1 expression and the effectiveness of immune-induced host defense against the tumor measured by TILs and prognosis of patients [26-28]. In this study, we observed that the higher density of TILs was associated with high PD-L1 expression in tumor cells of OSCC. Consistent with our results, previous studies reported the correlation between PDL1 expression of tumor and microenvironmental TILs in cancers of the colon, breast, larynx, and thymus [2831]. Moreover, studies that detected the PD-L1 mRNA expression showed that high PD-L1 expression is associated with increased TILs and favorable prognosis, as shown in our study [29-32]. These results seem paradoxical, given the inhibitory role of PD-L1 in immune responses. Considering the conflicting results of the PD-L1 association with TILs and prognosis according to tumor types or even within the same type of tumors [27, 33, 34], PD-L1 expression of tumor or recruited number of TILs might not be a surrogate marker for the final status of effective immune evasion, but a marker of active immune response by tumor cells to evade anti-tumor immune attack by host TILs, regardless of its effectiveness. Therefore, high PD-L1 expression and accumulated TILs in our study might reflect unsuccessfully skewed immune evasion [35], which remains to be clarified further.

In the present study of OSCC, high miR-197 was associated with higher tumor stage (T4) and poor prognosis, whereas high PD-L1 expression in tumors was associated with good prognosis. Although TILs did not directly correlate with survival of OSCC patients, low numbers of various TILs were associated with aggressive features of tumors, including high stage, angiolymphatic invasion, and perineural invasion. The phase of anti-tumor immune reaction might be different between early tumors and full-blown tumors because of different exposure time and amount of tumor antigens, and the mechanism of associations between miR-197/ PD-L1/TILs and stages of disease must be investigated further. Considering that many clinicopathologic factors and outcomes can influence or be influenced by antitumor immune reactions [36], comprehensive analyses of miR-197/PD-L1 and TILs could be more useful than individual analysis of PD-L1. Studies suggest that the miRNA is a factor in controlling the PD-1/PDL1 signaling for cancer immunotherapy [18, 37]. Our approach with miR-197, PD-L1, and TILs could be effective in recognizing the clinicopathologic implication of miR-197/PD-L1 and TILs, and selecting patients for immunotherapeutic strategy and avoiding immunerelated adverse reactions in OSCC.

Studies related to the expression of PD-L1 and its prognostic effects in different cancer types show discordant results [38-40]. Various clinicopathologic factors, including cancer types and individual host factors could influence pattern, degree, and effectiveness of anti-tumor immune reactions. Furthermore, different methods of detecting PD-L1 protein or mRNA have been developed, including IHC that utilizes various antibodies [41], in situ hybridization assay [32], DNA microarrays [29], and quantitative real-time PCR [30, 31]. In addition, pre-operative neoadjuvant treatment might influence expression of PD-L1, as well as miR-197 and TILs. In this study, neoadjuvant treatment was administered in only $4.4 \%$ of patients, with no significant correlation with clinicopathologic variables, suggesting limited effects of neoadjuvant treatments. Because these various factors and techniques might make the results of PD-L1 expression widely variable, previous clinicopathologic studies must be interpreted carefully [42]. In this context, our miR-197/PD-L1-paired approach can contribute to understanding the mechanism and clinical significance of PD-L1-associated anti-tumor host immune function in OSCC. 
This study showed inverse correlation and prognostic effects between miR-197 and PD-L1 expression in OSCC. In addition, we observed that PDL1 expression on IHC is associated with increased TILs and favorable prognosis in miR-197 $7^{\text {high }}$ subgroup. Our clinicopathologic analysis of miR-197/PD-L1 and TILs could contribute to understanding of clinical significance of anti-tumor immune reactions in OSCC.

\section{MATERIALS AND METHODS}

\section{Patients, samples, clinical data, and study design}

A total of 68 OSCC patients who underwent curative surgery between 2003 and 2011 at Seoul National University Bundang Hospital were enrolled. We reviewed hematoxylin-eosin (H\&E), immunohistochemically stained slides, pathology reports, and medical records to establish the clinicopathologic features of the tumors. TNM staging was determined according to the seventh edition of the American Joint Committee on Cancer guidelines [43]. Tissue microarrays were constructed by collecting single-tissue cores ( $2 \mathrm{~mm}$ in diameter) from the most morphologically representative areas of formalin-fixed, paraffin-embedded (FFPE) tissue specimens, as previously described [44]. In the analysis of the clinical data, disease-free survival (DFS) was defined as survival time from curative surgical resection to the last follow-up date of diseasefree state or to death. Overall survival (OS) was defined as survival time from curative surgical resection to death by any cause. The time intervals for the regular followup was 3 months after surgery for the first 2 years, and then every 6 months for the next 3 years. The followup period changed if the patient had symptoms or signs that possibly correlated with disease or treatment at the regular visit. Mean follow-up duration was 44.3 months (range, 2.1-122.0 months). During the follow-up period, $28 \%(19 / 68)$ of patients had recurrence and $34 \%(23 / 68)$ of patients died. The remainder of the patients were considered censored in survival analysis for DFS and OS. The primary objective was to determine the correlation among miR-197, PD-L1, TILs, and clinicopathologic factors. The secondary objective was to determine the prognostic effects of miR-197, PD-L1, and TILs on OS and DFS.

\section{RNA extraction and quantitative PCR for miR- 197 expression analysis}

Total RNA was extracted from $10-\mu$ m-thick FFPE tissue sections by use of a RecoverAll Total Nucleic Acid Isolation Kit for FFPE samples (Applied Biosystems, Foster City, CA, USA), and stored at $-80^{\circ} \mathrm{C}$ until the time of use after measuring the concentration with a NanoDrop 2000 spectrophotometer (Thermo Fisher
Scientific, Waltham, MA, USA). To measure the relative expression level of hsa-miR-197 (Catalog no. \#4427975, Applied Biosystems), reverse transcription and real-time polymerase chain reaction were conducted by use of $10 \mathrm{ng}$ of total RNA, Universal PCR Master Mix and a TaqMan microRNA Reverse Transcription kit (Catalog no. 4366596, Applied Biosystems) with U6 snRNA as an internal reference gene and reactive tonsil tissue as a normal control, as previously described [45]. The relative level of miR-197 in OSCC was calculated as $2^{-\Delta \Delta \mathrm{Ct}}$, where $\Delta \mathrm{Ct}=\mathrm{Ct}(\mathrm{miR}-197)-\mathrm{Ct}$ (U6) and $\Delta \Delta \mathrm{Ct}=\Delta \mathrm{Ct}$ (tumor) $\Delta \mathrm{Ct}$ (normal).

\section{Immunohistochemistry}

Immunohistochemistry (IHC) staining was applied to TMA sections $(4 \mu \mathrm{m})$ by use of the Ventana Benchmark XT automated staining system (Ventana Medical Systems, Tucson, AZ, USA). The following primary antibodies were used: polyclonal rabbit anti-human PDL1 (clone ab153991, Abcam, Cambridge, UK; 1:1000), monoclonal rabbit anti-human PD-1 (clone \#6796-1, Epitomics, Burlingame, CA, USA; 1:300), monoclonal rabbit anti-human CD4 (clone SP35, Catalog no. 7904423, Ventana Medical Systems), monoclonal mouse antihuman CD8 (clone C8/144B, Catalog no. IR623, Dako, Carpinteria, CA, USA), mouse monoclonal anti-human FoxP3 (clone \#236A/E7, Catalog no. ab20034, Abcam; 1:50), rabbit monoclonal anti-human CD3 (clone \#SP7, Catalog no. RM-9107-S, Thermo Scientific, Waltham, MA, USA; 1:100), mouse monoclonal anti-human CD20 (clone \#L26, Catalog no. IR604, Dako). Chorionic villi of human placenta were used as positive control, and human skin tissue was used as negative control. In this setting, we adjusted the staining condition for human oral cancer tissues. Immunostaining was interpreted by two pathologists (H.A. and J.H.P.). PD-L1 was stained in cytoplasm or cell membrane of tumor cells or both and graded as $0,1+$ and $2+$, according to the intensity of PDL1.

\section{Interpretation of immunohistochemical staining}

All immunostained slides were blindly evaluated by two experienced pathologists (H.A. and J.H.P.). PDL1 IHC was defined as membranous or by cytoplasmic positivity and scored as follows: 0 indicates no staining or staining in $<10 \%$ of the tumor cells, $1+$ indicates staining in $\geq 10 \%$ of the tumor cells with weak positivity, and $2+$ indicates moderate to strong positivity, which was modified from several previous studies [46-48]. The numbers of $\mathrm{CD} 3+, \mathrm{CD} 4+, \mathrm{CD} 8+, \mathrm{PD}-1+$, FoxP3+, and CD20+ TILs around the tumor cells were counted in three representative areas under a high-power optical microscope (400x). The average absolute number was recorded. 


\section{Statistical analysis}

Statistical analysis was done mainly by SPSS Statistic Version 21.0 software package (IBM Corp., Armonk, NY, USA). Chi-square tests, Student's $t$-test, Pearson's correlation test, and Spearman's rho test were used to examine the relations among expression of miR197, PD-L1, number of TILs, and clinicopathologic variables. Survival analysis was performed by use of the Cox regression hazard model. For the multivariate survival analysis, clinicopathologic variables with significant $P$ values $(<0.05)$ in univariate analysis were entered into the multivariate Cox model, and $P$-values of $<0.05$ were considered significant. R packages (Version 3.1.2) (http:// www.r-project.org) were used in the graphical dot-plot analysis [49-51].

\section{ACKNOWLEDGMENTS}

This work was supported by a grant (02-2013-022) from the Seoul National University Bundang Hospital Research Fund.

\section{CONFLICTS OF INTERESTS}

The authors declare no competing financial interests.

\section{REFERENCES}

1. Torre LA, Sauer AM, Chen MS Jr, Kagawa-Singer M, Jemal A, Siegel RL. Cancer statistics for Asian Americans, Native Hawaiians, and Pacific Islanders, 2016: converging incidence in males and females. CA Cancer J Clin. 2016; 66: 182-202.

2. Chi AC, Day TA, Neville BW. Oral cavity and oropharyngeal squamous cell carcinoma--an update. CA Cancer J Clin. 2015; 65: 401-21.

3. Salian V, Dinakar C, Shetty P, Ajila V. Etiological trends in oral squamous cell carcinoma: a retrospective institutional study. Cancer TranslMed. 2016; 2: 33-6.

4. Liviu Feller JL. Oral squamous cell carcinoma: epidemiology, clinical presentation and treatment. J Cancer Ther. 2012; 3: 263-8.

5. Okazaki T, Honjo T. PD-1 and PD-1 ligands: from discovery to clinical application. Int Immunol. 2007; 19: 813-24.

6. Zou W, Wolchok JD, Chen L. PD-L1 (B7-H1) and PD-1 pathway blockade for cancer therapy: mechanisms, response biomarkers, and combinations. Sci Transl Med. 2016; 8: 328rv4.

7. Cho YA, Yoon HJ, Lee JI, Hong SP, Hong SD. Relationship between the expressions of PD-L1 and tumor-infiltrating lymphocytes in oral squamous cell carcinoma. Oral Oncol. 2011; 47: 1148-53.

8. Zandberg DP, Strome SE. The role of the PD-L1:PD-1 pathway in squamous cell carcinoma of the head and neck. Oral Oncol. 2014; 50: 627-32.
9. Manikandan M, Deva Magendhra Rao AK, Arunkumar G, Manickavasagam M, Rajkumar KS, Rajaraman R, Munirajan AK. Oral squamous cell carcinoma: microRNA expression profiling and integrative analyses for elucidation of tumourigenesis mechanism. Mol Cancer. 2016; 15: 28.

10. Soga D, Yoshiba S, Shiogama S, Miyazaki H, Kondo S, Shintani S. microRNA expression profiles in oral squamous cell carcinoma. Oncol Rep. 2013; 30: 579-83.

11. Mavridis K, Gueugnon F, Petit-Courty A, Courty Y, Barascu A, Guyetant S, Scorilas A. The oncomiR miR-197 is a novel prognostic indicator for non-small cell lung cancer patients. Br J Cancer. 2015; 112: 1527-35.

12. Fujita Y, Yagishita S, Hagiwara K, Yoshioka Y, Kosaka N, Takeshita F, Fujiwara T, Tsuta K, Nokihara H, Tamura T, Asamura H, Kawaishi M, Kuwano K, et al. The clinical relevance of the miR-197/CKS1B/STAT3-mediated PD-L1 network in chemoresistant non-small-cell lung cancer. Mol Ther. 2015; 23: 717-27.

13. Chen J, Jiang CC, Jin L, Zhang XD. Regulation of PD-L1: a novel role of pro-survival signalling in cancer. Ann Oncol. 2016; 27: 409-16.

14. Zhao L, Yu H, Yi S, Peng X, Su P, Xiao Z, Liu R, Tang A, Li $X$, Liu F, Shen S. The tumor suppressor miR-138-5p targets PD-L1 in colorectal cancer. Oncotarget. 2016; 7: 45370-84. doi: 10.18632/oncotarget.9659.

15. Gong AY, Zhou R, Hu G, Li X, Splinter PL, O'Hara SP, LaRusso NF, Soukup GA, Dong H, Chen XM. MicroRNA-513 regulates $\mathrm{B} 7-\mathrm{H} 1$ translation and is involved in IFN-gamma-induced B7-H1 expression in cholangiocytes. J Immunol. 2009; 182: 1325-33.

16. Wang W, Li F, Mao Y, Zhou H, Sun J, Li R, Liu C, Chen W, Hua D, Zhang X. A miR-570 binding site polymorphism in the B7-H1 gene is associated with the risk of gastric adenocarcinoma. Hum Genet. 2013; 132: 641-8.

17. Wang X, Li J, Dong K, Lin F, Long M, Ouyang Y, Wei J, Chen X, Weng Y, He T, Zhang H. Tumor suppressor miR-34a targets PD-L1 and functions as a potential immunotherapeutic target in acute myeloid leukemia. Cell Signal. 2015; 27: 443-52.

18. Chen L, Gibbons DL, Goswami S, Cortez MA, Ahn YH, Byers LA, Zhang X, Yi X, Dwyer D, Lin W, Diao L, Wang J, Roybal JD, et al. Metastasis is regulated via microRNA-200/ZEB1 axis control of tumour cell PD-L1 expression and intratumoral immunosuppression. Nat Commun. 2014; 5: 5241.

19. Martin-Ezquerra G, Salgado R, Toll A, Baro T, Mojal S, Yebenes M, Garcia-Muret MP, Sole F, Quitllet FA, Espinet B, Pujol RM. CDC28 protein kinase regulatory subunit 1B (CKS1B) expression and genetic status analysis in oral squamous cell carcinoma. Histol Histopathol. 2011; 26: 71-7.

20. Kitajima S, Kudo Y, Ogawa I, Bashir T, Kitagawa M, Miyauchi M, Pagano M, Takata T. Role of Cks1 overexpression in oral squamous cell carcinomas: cooperation with Skp2 in promoting p27 degradation. Am J Pathol. 2004; 165: 2147-55. 
21. Peyser ND, Pendleton K, Gooding WE, Lui VW, Johnson DE, Grandis JR. Genomic and transcriptomic alterations associated with STAT3 activation in head and neck cancer. PLoS One. 2016; 11: e0166185.

22. Dai W, Wang C, Wang F, Wang Y, Shen M, Chen K, Cheng P, Zhang Y, Yang J, Zhu R, Zhang H, Li J, Zheng Y, et al. Anti-miR-197 inhibits migration in HCC cells by targeting KAI 1/CD82. Biochem Biophys Res Commun. 2014; 446: 541-8.

23. Keutgen XM, Filicori F, Crowley MJ, Wang Y, Scognamiglio T, Hoda R, Buitrago D, Cooper D, Zeiger MA, Zarnegar R, Elemento O, Fahey TJ 3rd. A panel of four miRNAs accurately differentiates malignant from benign indeterminate thyroid lesions on fine needle aspiration. Clin Cancer Res. 2012; 18: 2032-8.

24. Ritprajak P, Azuma M. Intrinsic and extrinsic control of expression of the immunoregulatory molecule PD-L1 in epithelial cells and squamous cell carcinoma. Oral Oncol. 2015; 51: 221-8.

25. Concha-Benavente F, Srivastava RM, Trivedi S, Lei Y, Chandran U, Seethala RR, Freeman GJ, Ferris RL. Identification of the cell-intrinsic and -extrinsic pathways downstream of EGFR and IFNgamma that induce PD-L1 expression in head and neck cancer. Cancer Res. 2016; 76: 1031-43.

26. Kim MY, Koh J, Kim S, Go H, Jeon YK, Chung DH. Clinicopathological analysis of PD-L1 and PD-L2 expression in pulmonary squamous cell carcinoma: comparison with tumor-infiltrating $\mathrm{T}$ cells and the status of oncogenic drivers. Lung Cancer. 2015; 88: 24-33.

27. Ameratunga M, Asadi K, Lin X, Walkiewicz M, Murone C, Knight S, Mitchell P, Boutros P, John T. PD-L1 and tumor infiltrating lymphocytes as prognostic markers in resected NSCLC. PLoS One. 2016; 11: e0153954.

28. Li Y, Liang L, Dai W, Cai G, Xu Y, Li X, Li Q, Cai S. Prognostic impact of programed cell death-1 (PD-1) and PD-ligand 1 (PD-L1) expression in cancer cells and tumor infiltrating lymphocytes in colorectal cancer. Mol Cancer. 2016; 15: 55.

29. Sabatier R, Finetti P, Mamessier E, Adelaide J, Chaffanet M, Ali HR, Viens P, Caldas C, Birnbaum D, Bertucci F. Prognostic and predictive value of PDL1 expression in breast cancer. Oncotarget. 2015; 6: 5449-64. doi: 10.18632/ oncotarget.3216.

30. Vassilakopoulou M, Avgeris M, Velcheti V, Kotoula V, Rampias T, Chatzopoulos K, Perisanidis C, Kontos CK, Giotakis AI, Scorilas A, Rimm D, Sasaki C, Fountzilas $\mathrm{G}$, et al. Evaluation of PD-L1 expression and associated tumor-infiltrating lymphocytes in laryngeal squamous cell carcinoma. Clin Cancer Res. 2016; 22: 704-13.

31. Yokoyama S, Miyoshi H, Nakashima K, Shimono J, Hashiguchi T, Mitsuoka M, Takamori S, Akagi Y, Ohshima K. Prognostic value of programmed death ligand 1 and programmed death 1 expression in thymic carcinoma. Clin Cancer Res. 2016.
32. Schalper KA, Velcheti V, Carvajal D, Wimberly H, Brown J, Pusztai L, Rimm DL. In situ tumor PD-L1 mRNA expression is associated with increased TILs and better outcome in breast carcinomas. Clin Cancer Res. 2014; 20 : 2773-82.

33. Schmidt LH, Kummel A, Gorlich D, Mohr M, Brockling S, Mikesch JH, Grunewald I, Marra A, Schultheis AM, Wardelmann E, Muller-Tidow C, Spieker T, Schliemann $\mathrm{C}$, et al. PD-1 and PD-L1 expression in NSCLC indicate a favorable prognosis in defined subgroups. PLoS One. 2015; 10: e0136023.

34. Tokito T, Azuma K, Kawahara A, Ishii H, Yamada K, Matsuo N, Kinoshita T, Mizukami N, Ono H, Kage M, Hoshino T. Predictive relevance of PD-L1 expression combined with CD8+ TIL density in stage III nonsmall cell lung cancer patients receiving concurrent chemoradiotherapy. Eur J Cancer. 2016; 55: 7-14.

35. Schalper KA. PD-L1 expression and tumor-infiltrating lymphocytes: revisiting the antitumor immune response potential in breast cancer. Oncoimmunology. 2014; 3: e29288.

36. Wimberly H, Brown JR, Schalper K, Haack H, Silver MR, Nixon C, Bossuyt V, Pusztai L, Lannin DR, Rimm DL. PD-L1 expression correlates with tumor-infiltrating lymphocytes and response to neoadjuvant chemotherapy in breast cancer. Cancer Immunol Res. 2015; 3: 326-32.

37. Chinai JM, Janakiram M, Chen F, Chen W, Kaplan M, Zang X. New immunotherapies targeting the PD-1 pathway. Trends Pharmacol Sci. 2015; 36: 587-95.

38. Darb-Esfahani S, Kunze CA, Kulbe H, Sehouli J, Wienert S, Lindner J, Budczies J, Bockmayr M, Dietel M, Denkert C, Braicu I, Johrens K. Prognostic impact of programmed cell death-1 (PD-1) and PD-ligand 1 (PD-L1) expression in cancer cells and tumor-infiltrating lymphocytes in ovarian high grade serous carcinoma. Oncotarget. 2016; 7: 1486-99. doi: 10.18632/oncotarget.6429.

39. Cooper WA, Tran T, Vilain RE, Madore J, Selinger CI, Kohonen-Corish M, Yip P, Yu B, O’Toole SA, McCaughan BC, Yearley JH, Horvath LG, Kao S, et al. PD-L1 expression is a favorable prognostic factor in early stage non-small cell carcinoma. Lung Cancer. 2015; 89: 181-8.

40. Gao Q, Wang XY, Qiu SJ, Yamato I, Sho M, Nakajima Y, Zhou J, Li BZ, Shi YH, Xiao YS, Xu Y, Fan J. Overexpression of PD-L1 significantly associates with tumor aggressiveness and postoperative recurrence in human hepatocellular carcinoma. Clin Cancer Res. 2009; 15: 971-9.

41. Kerr KM, Hirsch FR. Programmed death ligand-1 immunohistochemistry: friend or Foe? Arch Pathol Lab Med. 2016; 140: 326-31.

42. Scognamiglio G, De Chiara A, Di Bonito M, Tatangelo F, Losito NS, Anniciello A, De Cecio R, D’Alterio C, Scala $\mathrm{S}$, Cantile M, Botti G. Variability in immunohistochemical detection of programmed death ligand 1 (PD-L1) in cancer tissue types. Int J Mol Sci. 2016; 17. 
43. Edge SB, Compton CC. The American Joint Committee on Cancer: the 7th edition of the AJCC cancer staging manual and the future of TNM. Ann Surg Oncol. 2010; 17: 1471-4.

44. Paik JH, Jeon YK, Park SS, Kim YA, Kim JE, Huh J, Lee SS, Kim WH, Kim CW. Expression and prognostic implications of cell cycle regulatory molecules, p16, p21, p27, p14 and p53 in germinal centre and nongerminal centre B-like diffuse large B-cell lymphomas. Histopathology. 2005; 47: 281-91.

45. Paik JH, Jang JY, Jeon YK, Kim WY, Kim TM, Heo DS, Kim CW. MicroRNA-146a downregulates NFkappaB activity via targeting TRAF6 and functions as a tumor suppressor having strong prognostic implications in NK/T cell lymphoma. Clin Cancer Res. 2011; 17: 4761-71.

46. Wang X, Teng F, Kong L, Yu J. PD-L1 expression in human cancers and its association with clinical outcomes. Onco Targets Ther. 2016; 9: 5023-39.
47. Meng X, Huang Z, Teng F, Xing L, Yu J. Predictive biomarkers in PD-1/PD-L1 checkpoint blockade immunotherapy. Cancer Treat Rev. 2015; 41: 868-76.

48. Choueiri TK, Figueroa DJ, Fay AP, Signoretti S, Liu Y, Gagnon R, Deen K, Carpenter C, Benson P, Ho TH, Pandite L, de Souza P, Powles T, et al. Correlation of PD-L1 tumor expression and treatment outcomes in patients with renal cell carcinoma receiving sunitinib or pazopanib: results from COMPARZ, a randomized controlled trial. Clin Cancer Res. 2015; 21: 1071-7.

49. Team RC. R: A language and environment for statistical computing. R Foundation for Statistical Computing. 2016.

50. Eklund A. beeswarm: the Bee Swarm Plot, an Alternative to Stripchart. R package version 0.2.3. 2016.

51. Wickham H. ggplot2: elegant graphics for data analysis. 2009. 